\title{
Prevalência de Diabetes Mellitus autorreferida entre trabalhadores de enfermagem*
}

\author{
Self-reported prevalence of Diabetes Mellitus among nursing workers
}

Prevalencia de Diabetes Mellitus autorreferida entre trabajadores de enfermería

\section{Cássia Andrade Martins ${ }^{1}$, Odete de Oliveira Monteiro ${ }^{2}$, Dulce Aparecida Barbosa $^{2}$, Ana Rita de Cássia Bettencourt ${ }^{2}$}

\section{RESUMO}

Objetivos: Verificar a prevalência de Diabetes Mellitus autorreferida em trabalhadores de enfermagem de um hospital universitário; identificar os principais fatores de risco para o Diabetes Mellitus (DM) e, entre os que já possuem a doença, identificar as dificuldades relacionadas a seu controle. Métodos: Estudo epidemiológico transversal, realizado no período de agosto de 2007 a janeiro de 2008 . Foram entrevistados 1.287 profissionais de enfermagem (82\%), utilizando-se questionário contendo 22 questões fechadas. A análise dos dados foi realizada pelos testes Qui-quadrado e exato de Fisher. O teste t de Student foi empregado para as variáveis contínuas. Resultados: Observou-se a prevalência de 3\% de DM autorreferida. Os principais fatores de risco reconhecidos foram: idade, sexo, peso e histórico familiar. Detectou-se que o plantão noturno concentrava mais da metade dos diabéticos. Conclusão: A prevalência de DM autorreferida nesse hospital foi menor do que a esperada para a cidade de São Paulo. Dentre os fatores de risco encontrados, o sobrepeso é o único modificável. O plantão noturno concentra a maioria dos funcionários com diabetes.

Descritores: Prevalência; Diabetes mellitus; Recursos humanos de enfermagem no hospital

\begin{abstract}
Objectives: To verify the self-reported prevalence of Diabetes Mellitus in nursing workers at a university hospital, to identify the main risk factors for diabetes mellitus (DM) and, among those who already have the disease, to identify the difficulties related to its control. Methods: This is a cross-sectional epidemiological study, conducted between August 2007 and January 2008. Of the total $(1,287)$ interviewed, 82\% were nurses. It was used a questionnaire containing 22 closed questions. The data analysis was performed with Chi-square test and Fisher exact test. Student $t$ test was used for continuous variables. Results: There was a prevalence of 3\% of self-reported DM. The main recognized risk factors were: age, sex, weight and, family history. It was found that the night shift contained more than half of diabetics. Conclusion: The self-reported prevalence of DM at the hospital was less than the expected value for the city of Sao Paulo. Among the risk factors found, the overweight was the only modifiable. The night shift concentrated most of the workers with diabetes.
\end{abstract}

Keywords: Prevalence; Diabetes mellitus; Nursing staff, hospital

\section{RESUMEN}

Objetivos: Verificar la prevalencia de Diabetes Mellitus autorreferida en trabajadores de enfermería de un hospital universitario; identificar los principales factores de riesgo para la Diabetes Mellitus (DM) y, entre los que ya poseen la enfermedad, identificar las dificultades relacionadas a su control. Métodos: Se trata de un estudio epidemiológico transversal, realizado en el período de agosto de 2007 a enero de 2008. Fueron entrevistados 1.287 profesionales de enfermería $(82 \%)$, utilizando un cuestionario que contenía 22 preguntas cerradas. El análisis de los datos fue realizado con las pruebas Chi-cuadrado y exacta de Fisher. La prueba t de Student fue empleada para las variables continuas. Resultados: Se observó una prevalencia de 3\% de DM autorreferida. Los principales factores de riesgo reconocidos fueron: edad, sexo, peso e histórico familiar. Se detectó que el plantón nocturno concentraba más de la mitad de los diabéticos. Conclusión: La prevalencia de DM autorreferida en ese hospital fue menor que la esperada para la ciudad de Sao Paulo. Entre los factores de riesgo encontrados, el sobrepeso es el único modificable. El plantón nocturno concentra la mayoría de los funcionarios con diabetes.

Descritores: Prevalencia; Diabetes mellitus; Personal de enfermería en hospital

* Estudo desenvolvido no Hospital São Paulo da Universidade Federal de São Paulo - UNIFESP - São Paulo (SP), Brasil.

1 Acadêmica de enfermagem da Universidade Federal de São Paulo - UNIFESP - São Paulo (SP), Brasil.

${ }^{2}$ Doutora em Enfermagem. Professor Adjunto do Departamento de Enfermagem da Universidade Federal de São Paulo - UNIFESP - São Paulo (SP), Brasil. 


\section{INTRODUÇÃO}

As doenças crônicas não transmissíveis, como Diabetes Mellitus (DM), hipertensão arterial e câncer, compõem um grupo de entidades que se caracterizam por apresentar, de forma geral, prolongado tempo de evolução, longo período de latência, lesões irreversíveis e complicações que acarretam graus variáveis de incapacidade ou óbito. Essas doenças vêm ocupando um maior espaço no perfil de morbidade e mortalidade das populações latinoamericanas $^{(1)}$.

Estudos do Banco Mundial demonstram que as doenças crônicas não transmissíveis são responsáveis por uma taxa cinco a nove vezes maior de morte prematura do que as doenças transmissíveis, bem como taxas de dez a cinco vezes maiores de incapacidade, em homens e mulheres, respectivamente ${ }^{(2)}$. Nos países em desenvolvimento, o DM já adquiriu características de epidemia. O Brasil ocupa o oitavo lugar entre os países com maior prevalência de DM. Em 2025, calcula-se que possam existir cerca de 11 milhões de pessoas diabéticas no País ${ }^{(2)}$.

Os fatores de risco para o desenvolvimento do DM, especialmente, o tipo 2 podem ser classificados em não modificáveis e modificáveis. Dentre os não modificáveis, podemos citar: idade, história familiar e presença de diabetes gestacional prévia. Os fatores modificáveis são de grande importância, visto que podem ser alvos de intervenção, destacando-se: obesidade, alimentação, sedentarismo, tabagismo e estresse psicossocial ${ }^{(3)}$.

A Federação Internacional de Diabetes criou uma estratégia baseada na prevenção de DM tipo 2 por meio do controle de fatores de risco modificáveis. Para aqueles indivíduos com alto risco de desenvolver a doença, criouse um plano composto por três etapas: identificação das pessoas com alto risco para DM, utilizando- se um questionário semiestruturado, mensuração do nível de risco e intervenção para diminuir os fatores de risco ${ }^{(4)}$.

Estudo randomizado controlado comprovou que a prevenção deve ser realizada pela prática de atividade física moderada durante $30-40 \mathrm{~min}$. na maioria dos dias da semana, associada à ingestão de alimentos ricos em fibras, com baixo teor calórico e baixo teor de gordura saturada ${ }^{(5)}$.

O DM tipo 1 é resultante da destruição das células betapancreáticas e tem como características início abrupto dos sintomas, facilidade para cetose e pouca influência hereditária, e o DM tipo 2 ocorre em razão da resistência insulínica. Este último é caracterizado pelo início insidioso, sintomas inespecíficos e forte componente hereditário( ${ }^{(6)}$.

O tratamento da doença deve ser individualizado, respeitando-se a idade, o estado mental, situação financeira e a cooperação do paciente. Basicamente, a terapia inicia-se com a mudança dos hábitos de vida pela manutenção do peso adequado, prática regular de atividade física, suspensão do hábito de fumar, baixa ingestão de gordura saturada, bem como de bebidas alcoólicas ${ }^{(6)}$. As pessoas com tipo 1 associam essas mudanças com a administração de insulina subcutânea e as com tipo 2, caso não atinjam o resultado esperado com as alterações nos hábitos diários, iniciam medicação complementar ${ }^{(6)}$.

As complicações da doença resultam em piora da qualidade de vida das pessoas que, com frequência, apresentam incapacidade parcial ou total, acarretando em perdas para o paciente, sua família e a sociedade, visto que a prevalência da doença entre a população adulta é de 7,6\%, comprometendo a saúde de grande parte da população economicamente ativa que, além de perder a produtividade no trabalho também se aposenta precocemente $^{(7)}$.

Os custos diretos para o atendimento ao paciente com DM no mundo variam de $2,5 \%$ a $15 \%$ dos gastos nacionais em saúde, dependendo da prevalência local da doença e da complexidade do tratamento disponível. Outros custos associados com a dor, ansiedade, inconveniência e menor qualidade de vida também merecem ser $\operatorname{citados}^{(7)}$.

A identificação da prevalência de DM entre uma população específica é fundamental para o planejamento das ações de prevenção focalizadas nos principais fatores de risco da doença, bem como a ampliação e melhora da qualidade do atendimento prestado a essa população. A intervenção precoce sobre pessoas que apresentam DM, além de aumentar a expectativa e qualidade de vida, minimizam os gastos públicos com saúde ${ }^{(7)}$.

Um dos princípios de cuidar cita que a forma como nos cuidamos está ligada à condição em que cuidamos do outro, "temos que tratar de nós, antes de podermos respeitar e cuidar dos outros"(8). Portanto, o autocuidado por parte do cuidador é fator essencial para se atingir uma assistência de qualidade. O profissional de saúde sofre um desgaste físico e mental muito grande que, muitas vezes, interfere na prestação do cuidado ${ }^{(9)}$.

Sabe-se que a condição de saúde a qual o trabalhador de enfermagem está exposto irá refletir diretamente na qualidade do trabalho de toda equipe, visto que a doença eleva os índices de ausência de funcionários. Na enfermagem, o absenteísmo é preocupante, pois desorganiza o serviço, gera insatisfação e sobrecarga entre os trabalhadores presentes e, consequentemente, diminui a qualidade da assistência prestada ao paciente ${ }^{(10)}$.

A Health Education Authority classificou a enfermagem, como a quarta profissão mais estressante no setor público. Para as autoras, elementos como o número reduzido de profissionais, o excesso de atividades executadas e as dificuldades para delimitar os diferentes 
papéis entre auxiliares, técnicos de enfermagem e enfermeiros são ameaçadores ao meio ambiente ocupacional do enfermeiro ${ }^{(11)}$. A prevenção de agravos à saúde dos trabalhadores de enfermagem é uma prioridade na atualidade; por isso, as condições de trabalho e saúde desses profissionais vêm sendo estudadas progressivamente em todo o mundo ${ }^{(12)}$.

Diante do exposto, traçamos os objetivos do presente estudo: verificar a prevalência de DM em trabalhadores de enfermagem de um hospital universitário; identificar os principais fatores de risco para o DM e, entre os que já possuem a doença, identificar as dificuldades relacionadas a seu controle.

\section{MÉTODOS}

Estudo epidemiológico, transversal. Após aprovação do projeto pelo Comitê de Ética em Pesquisa da Universidade Federal de São Paulo, Protocolo n. ${ }^{\circ}$ 1517/ 07, a pesquisa foi realizada em um hospital universitário, localizado na zona sul da cidade de São Paulo, entre agosto de 2007 a janeiro de 2008. A população do estudo constituiu-se de 1570 trabalhadores de enfermagem que atuavam nessa instituição no período da coleta de dados. Foram incluídos, no estudo, 1287 (82\%) funcionários presentes nesse período e que concordaram em assinar o Termo De Consentimento Livre e Esclarecido (TCLE). Entre os 283 não participantes do estudo, 278 estavam de férias e/ou licença médica e cinco recusaram-se a participar.

As variáveis exploratórias foram: variáveis demográficas: sexo, idade, escolaridade e categoria profissional; variáveis relacionadas aos fatores de risco: Índice de Massa Corporal (IMC), histórico familiar, atividade física e hábito de fumar; e variáveis relacionadas à doença: tipo de $\mathrm{DM}$, tempo da doença, frequência à consulta médica, uso de medicamentos, orientação sobre a dieta, automonitorização no domićlíio e número de internações em decorrência do mau controle glicêmico. Todos os dados coletados, exceto o IMC, foram referidos pelos participantes deste estudo.

A variável atividade física foi considerada adequada quando praticada de 30 a 40 minutos, no mínimo três vezes por semana ${ }^{(5)}$.

A variável IMC foi analisada, conforme a Organização Mundial da Saúde ${ }^{(13)}$ que delimita valores para peso normal/ eutrófico $\left(18,5-24,9 \mathrm{~kg} / \mathrm{m}^{2}\right)$, baixo peso (< $18,5)$, sobrepeso $\left(25-29,9 \mathrm{~kg} / \mathrm{m}^{2}\right)$ e obeso $(30->=40$ $\left.\mathrm{kg} / \mathrm{m}^{2}\right)$.

Para a coleta de dados, utilizou-se um questionário com 22 perguntas fechadas. Após serem orientados sobre a pesquisa e assinarem o TCLE, os participantes respondiam o questionário, na presença do pesquisador.

A análise dos dados foi realizada por meio de teste
Qui-quadrado ou teste exato de Fisher; para relação entre as variáveis categóricas e para as variáveis quantitativas, utilizou-se o teste t de Student. Como parte da análise univariada foi avaliada a força da associação de cada uma das variáveis com a presença de diabetes pelo calculo do "odds ratio" (OR) e seu respectivo intervalo de confiança a 95\% (IC 95\%). Para a análise multivariada, foi ajustado o modelo de regressão logística, no qual foram introduzidas todas as variáveis que na análise univariada apresentaram p-valor menor do que 20\% $(\mathrm{p}<0,20)$. O modelo final foi constituído por todas as variáveis que se mostraram significantes.

A análise estatística foi realizada no "software" SPSS 16.0 for Windows (SPSS Inc.), o nível de significância (á) adotado foi de $5 \%$, ou seja, foram considerados como estatisticamente significantes os resultados que apresentaram $\mathrm{p}$-valor $<5 \%(\mathrm{p}<0,05)$.

\section{RESULTADOS}

A média de idade dos 1.287 funcionários foi de 37 anos (desvio-padrão $=10$ anos), variando de 19 a 69 anos. O sexo feminino foi o mais freqüente, representando, quase $85 \%$ do total de trabalhadores. A categoria mais frequente foi auxiliar de enfermagem (51\%), seguida de técnico em enfermagem $(25 \%)$ e enfermeiro (24\%). A escolaridade mais frequente foi o ensino médio (61\%) seguidos por ensino superior (36\%) e ensino fundamental (3\%). Quanto ao IMC, observouse que, aproximadamente, $52 \%$ dos funcionários foram classificados, como eutróficos, $2 \%$ como abaixo do peso, $30 \%$ como sobrepeso e $16 \%$ como obesos.

A prevalência de DM autorreferida nos trabalhadores de enfermagem foi igual a 3\% (IC 95\% [2,2\%; 4,1\%]), ou seja, 38 funcionários, desses, $79 \%$ referiram ter DM tipo 2 e $21 \%$, DM tipo 1 . Com relação ao turno de trabalho, $20(52,6 \%)$ eram do plantão noturno, 10 $(26,3 \%)$, do plantão da tarde e $8(21,1 \%)$, do plantão da manhã.

No dados Tabela 1, pode-se observar as características da população do estudo no grupo com DM autorreferida e no grupo sem DM autorreferida. Foi avaliada a influência isolada de cada variável na presença de DM sem levar em consideração as demais variáveis (análise univariada).

Dando continuidade à análise, foi utilizada a abordagem multivariada. Nesse ajuste, optou-se pela inclusão no modelo multivariado das variáveis que apresentaram $\mathrm{p}$ valor menor do que $20 \%(p<0,20)$ na análise univariada. Nos dados da Tabela 2, pode-se observar que idade, sexo, IMC e história familiar mostraram-se independentemente associadas a DM $(p<0,05)$.

Nos dados da Tabela 3, encontram-se as características relacionadas ao controle de DM. 
Tabela 1 - Caracterização dos profissionais de enfermagem de um hospital universitário, segundo presença ou não de DM. São Paulo, 2008

\begin{tabular}{|c|c|c|c|c|}
\hline & $\mathbf{D M}_{\text {referida }}$ & Não $\mathbf{D M}_{\text {referida }}$ & OR [IC 95\%] & Valor de $p$ \\
\hline Idade (anos) & $46,4 \pm 12,0$ & $36,4 \pm 10,1$ & $\begin{array}{c}1,085 \\
{[1,054-1,117]}\end{array}$ & $<0,001$ \\
\hline Sexo & & & & 0,060 \\
\hline Masculino & $10(26,3 \%)$ & $189(15,1 \%)$ & $\begin{array}{c}2,003 \\
{[0,957-4,192]}\end{array}$ & \\
\hline Feminino & $28(73,7 \%)$ & $1060(84,9 \%)$ & 1,000 & \\
\hline Peso (kg) & $77,3 \pm 15,8$ & $67,2 \pm 14,1$ & $\begin{array}{c}1,041 \\
{[1,021-1,061]}\end{array}$ & $<0,001$ \\
\hline $\operatorname{IMC}\left(\mathrm{kg} / \mathrm{m}^{2}\right)$ & $29,3 \pm 6,3$ & $25,1 \pm 4,6$ & $\begin{array}{c}1,155 \\
{[1,093-1,219]}\end{array}$ & $<0,001$ \\
\hline $\operatorname{IMC}\left(\mathrm{kg} / \mathrm{m}^{2}\right)$ & & & & 0,001 \\
\hline $\begin{array}{l}\text { Eutrófico } \\
\text { Baixo Peso }\end{array}$ & $\begin{array}{c}10(26,3 \%) \\
-\end{array}$ & $\begin{array}{c}656(52,5 \%) \\
34(2,7 \%)\end{array}$ & $\begin{array}{c}1,000 \\
\text { Não estimável }\end{array}$ & \\
\hline Sobrepeso & $13(34,2 \%)$ & $372(29,8 \%)$ & $\begin{array}{c}2,292 \\
{[0,995-5,279]}\end{array}$ & \\
\hline Obeso & $15(39,5 \%)$ & $187(15,0 \%)$ & $\begin{array}{c}5,262 \\
{[2,326-11,905]}\end{array}$ & \\
\hline Escolaridade & & & & 0,001 \\
\hline Fundamental & $4(10,5 \%)$ & $37(3,0 \%)$ & $\begin{array}{c}10,032 \\
{[2,583-38,961]}\end{array}$ & \\
\hline Médio & $29(76,3 \%)$ & $748(59,9 \%)$ & $\begin{array}{c}3,598 \\
{[1,383-9,360]}\end{array}$ & \\
\hline Superior & $5(13,2 \%)$ & $464(37,1 \%)$ & 1,000 & \\
\hline Categoria & & & & 0,126 \\
\hline Auxiliar & $24(63,2 \%)$ & $629(50,4 \%)$ & $\begin{array}{c}2,900 \\
{[0,997-8,431]}\end{array}$ & \\
\hline Técnico & $10(26,3 \%)$ & $316(25,3 \%)$ & $\begin{array}{c}2,405 \\
{[0,746-7,750]}\end{array}$ & \\
\hline Enfermeiro & $4(10,5 \%)$ & $304(24,3 \%)$ & 1,000 & \\
\hline Tabagismo & & & & 0,981 \\
\hline Sim & $5(13,2 \%)$ & $166(13,3 \%)$ & $\begin{array}{c}0,988 \\
{[0,381-2,568]}\end{array}$ & \\
\hline $\begin{array}{l}\text { Não } \\
\text { História familiar }\end{array}$ & $33(86,8 \%)$ & $1083(86,7 \%)$ & 1,000 & 0,001 \\
\hline 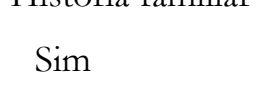 & $28(73,7 \%)$ & $575(46,0 \%)$ & $\begin{array}{c}3,282 \\
{[1,581-6,814]}\end{array}$ & \\
\hline Não & $10(26,3 \%)$ & $674(54,0 \%)$ & & \\
\hline Atividade física & & & & 0,444 \\
\hline Sim & $32\left(84,2^{\%} \%\right)$ & $988(79,1 \%)$ & $\begin{array}{c}1,409 \\
{[0,583-3,405]}\end{array}$ & \\
\hline Não & $6(15,8 \%)$ & $261(20,9 \%)$ & 1,000 & \\
\hline
\end{tabular}

Tabela 2: Fatores de risco para desenvolver DM, São Paulo, 2008

\begin{tabular}{lccc}
\hline & OR & {$[$ [C 95\%] } & Valor de $\boldsymbol{p}$ \\
\hline Idade (anos) & 1,088 & {$[1,053-1,123]$} & $<0,001$ \\
Sexo (Masculino x Feminino) & 2,926 & {$[1,321-6,482]$} & 0,008 \\
IMC (kg/m $)$ & 1,111 & {$[1,045-1,182]$} & 0,001 \\
História Familiar (Sim x Não) & 3,564 & {$[1,658-7,662]$} & 0,001 \\
\hline
\end{tabular}


Tabela 3: Controle da doença dos profissionais de enfermagem com DM autorreferida, São Paulo, 2008.

\begin{tabular}{lc}
\hline Quanto tempo DM (anos) & $5(1,5-10)$ \\
Mediana (Q1 - Q3) & $0,2-21$ \\
Mínimo - Máximo & \\
Faz tratamento com medicamento & $31(81,6 \%)$ \\
Sim & $7(18,4 \%)$ \\
Não & \\
Frequência de consultas & $2(6,5 \%)$ \\
Quinzenal & $2(6,5 \%)$ \\
Mensal & $8(25,8 \%)$ \\
Bimestral & $11(35,4 \%)$ \\
Trimestral & $8(25,8 \%)$ \\
Semestral & \\
Orientação de dieta & $36(94,7 \%)$ \\
Sim & $2(5,3 \%)$ \\
Não & \\
Segue & $20(52,6 \%)$ \\
Sim & $18(47,4 \%)$ \\
Não & \\
Automonitoramento & $28(73,7 \%)$ \\
Sim & $10(26,3 \%)$ \\
Não & \\
Internação & $8(21,1 \%)$ \\
Sim & $30(78,9 \%)$ \\
Não & \\
\hline
\end{tabular}

\section{DISCUSSÃO}

A prevalência de DM encontrada na cidade de São Paulo é classificada, como de nível intermediário (3\% a $10 \%)^{(14)}$. Estudo realizado em três bairros dessa cidade compostos por populações distintas, mostrou que metade dos sujeitos desconhecia a doença ${ }^{(15)}$; outro estudo realizado no Município de São Paulo, encontrou 4,7\% da população que referiram ter a doença; após a realização da glicemia capilar na mesma população, a porcentagem chegou a $9,7 \% \%^{(16)}$.

Verificamos que a prevalência de DM autorreferida entre os trabalhadores de enfermagem do hospital universitário foi de 3\%. Embora a amostra seja composta de indivíduos com idades entre 19 e 69 anos, os valores de prevalência encontrados foram abaixo dos descritos na literatura ${ }^{(14,17)}$. Estudo multicêntrico realizado, em 1987, mostrou que a prevalência da doença era de $7,6 \%$ na população de 30 a 69 anos $^{(17)}$.

O DM tipo 2 é característico da idade adulta, sua incidência e prevalência aumentam acentuadamente, com o progredir da idade, particularmente, após os $40 \operatorname{anos}^{(18)}$. Dentre os trabalhadores de enfermagem do hospital, a prevalência de DM revelou ser maior, conforme o aumento da idade: a cada ano somado à idade, a chance de se apresentar a doença aumenta em 9\% $(\mathrm{p}<0,001)$. Este crescimento de DM com a idade também foi encontrado em outro estudo ${ }^{(19)}$.

Não há tendência clara na literatura sobre a prevalência entre os sexos. O que se verifica é que essa freqüência constituise, mais do que por diferenciais genéticos, por um produto da presença de fatores de risco ${ }^{(14,16)}$. A análise da população estudada demonstra que a quantidade de trabalhadores com diabetes do sexo masculino supera em, aproximadamente, três vezes a do sexo feminino $(\mathrm{p}=0,008)$, comprovando a presença dessa variável, como fator de risco.

Sabe-se que a resistência insulínica é um importante elo de ligação entre a obesidade e a doença ${ }^{(20)}$. A obesidade configura-se, como característica marcante entre portadores de DM tipo 2 e os índices de IMC compatíveis com sobrepeso têm sido verificados ${ }^{(20)}$. Recentemente, pesquisa constatou que não há distinção entre medida de circunferência abdominal e IMC ao se identificar indivíduos com risco para desenvolver $\mathrm{DM}^{(21)}$.

No presente estudo, verificou-se quando o IMC aumenta em $1 \mathrm{~kg} / \mathrm{m}^{2}$, a chance do individuo apresentar DM aumenta em 11\% ( $p=0,001)$. O número de trabalhadores de enfermagem com diabetes e excesso de peso ultrapassa a marca dos $70 \%$, valor que não é diferente do encontrado na região Sudeste em estudo multicêntrico nacional sobre a prevalência de sobrepeso e obesidade entre os diabéticos do tipo 2 no Brasil ${ }^{20)}$.

O cigarro aumenta a concentração de gordura abdominal ${ }^{(22)}$, reduz a sensibilidade insulínica ${ }^{(23)}$ e eleva demasiadamente a concentração glicêmica ${ }^{(24)}$. A quantidade de cigarros e o tempo de tabagismo parecem estar relacionados ao risco de apresentar DM. Apesar da maior incidência da doença entre os fumantes ${ }^{(25)}$, na população estudada o tabagismo não se mostrou fator de risco significativo.

Pessoas com história familiar de DM tipo 2 têm maior tendência para desenvolver a doença. Além dos fatores comportamentais relacionados com o ambiente familiar, várias evidências vêm mostrando que parte do risco aumentado de desenvolvimento de DM é em razão de fatores genéticos, conforme ratificado num estudo que comprovou a presença de alterações genéticas em pessoas portadoras de $\mathrm{DM}^{(26)}$. Entre os trabalhadores que apresentaram DM, 73,7\% possuíam portadores da doença na família. Observou-se que a chance de um individuo com história familiar de diabetes apresentar DM é, quase, 3,6 vezes aquela observada entre indivíduos sem história familiar de diabetes $(\mathrm{p}=0,001)$. O resultado encontrado foi semelhante ao obtido em estudo realizado nos Estados Unidos da América, que observou a prevalência de DM em $73 \%$ dos participantes que possuíam pais com a doença ${ }^{(27)}$.

Estudo que objetivou relacionar o processo de trabalho com o adoecimento entre os enfermeiros identificou o estresse como principal fator de risco para o desenvolvimento de doenças crônicas, seguido de hipertensão arterial, obesidade, sedentarismo, DM, tabagismo e excesso de sal na alimentação ${ }^{(38)}$. 
A automonitorização está indicada para pacientes que fazem uso de medicação, tanto insulina como antidiabéticos orais ${ }^{(29)}$. Em pacientes com DM tipo 1 é recomendada a realização de três ou mais testes diários. Já os que apresentam DM tipo 2, a freqüência ótima de realização não está definida, mas depende do tipo do tratamento realizado (agentes orais ou insulina) e da estabilidade do quadro metabólico ${ }^{(30)}$.

Neste estudo, detectamos que, independente do tipo de diabetes, cerca de $26 \%$ dos trabalhadores de enfermagem não realizavam automonitorização com frequência diária, o que demonstra a vulnerabilidade destes indivíduos frente à descompensação da doença, bem como o comprometimento por parte das complicações que advêm da hiperglicemia.

Em relação ao tempo de diagnóstico, os diabéticos do tipo 1 demonstraram ter conhecimento há mais tempo (média de 11,6 anos) do que os do tipo 2 (média de 5,3 anos), dados estes que confirmam a cronicidade da doença.

O consumo alimentar constitui um dos principais fatores passíveis de modificação para o DM. Entre a população estudada, a maioria dos portadores do tipo 2 revelou que não costumava seguir a dieta adequadamente, embora tenha conhecimento dos hábitos alimentares considerados ideais para o controle da progressão da doença. Entre os trabalhadores com DM do tipo 1, o controle alimentar mostrou-se mais rigoroso, com adesão de $86 \%$ dos funcionários. Estudo que analisou as dificuldades do controle do DM refere que as principais razões citadas foram a dificuldade de controle dos impulsos e dificuldade de manter a dieta recomendada em razão dos hábitos adquiridos, horário definido, valor cultural do alimento, condições socioeconômicas e questão psicológica envolvida ${ }^{(30)}$.

Cabe ressaltar que, entre os profissionais responsáveis por orientar a dieta aos participantes do estudo, foram citados nutricionista e médico, mas a enfermeira não foi relacionada à prática dessa tarefa, o que demonstra uma lacuna no papel de cuidado da enfermagem, visto que a orientação por parte desses profissionais está relacionada a maiores índices de adesão ${ }^{(31)}$ e, portanto, imprescindível para o sucesso do tratamento.

Estimular a adesão ao tratamento é de suma importância para o controle da doença, sobretudo, por meio da educação em saúde, que deve abordar os aspectos psicológicos, culturais, sociais e interpessoais da pessoa com $\mathrm{DM}^{(32)}$. Apesar de se tratar de profissionais da área da saúde, grande parte não possui esclarecimento prévio sobre a doença, em especial aqueles indivíduos com menor nível de escolaridade.

O caráter assintomático da doença faz com que os pacientes, na maioria das vezes, não reconheçam a importância do tratamento medicamentoso e da dieta ${ }^{(33)}$. Nesta pesquisa, 22,6\% dos indivíduos que relataram ter DM do tipo 2 não realizavam acompanhamento da doença em serviços de saúde. Esse valor é considerável, conforme a doença causa comprometimentos a longo prazo, podendo até resultar em incapacitação de muitos portadores.

Em pesquisa realizada em um hospital universitário no interior do Paraná, descobriu -se que a cada dez faltas cometidas por profissionais de enfermagem, aproximadamente, sete são decorrentes de doença ${ }^{(10)}$.

A equipe de enfermagem realiza suas atividades divididas em três turnos diários, que compreendem os períodos matutino, vespertino e noturno. Este último caracteriza-se por 12 horas seguidas de trabalho. O organismo dos profissionais que trabalham à noite passa por um período de adaptação, visto que a espécie humana é diurna. A temperatura, os hormônios, a psique, o comportamento e o desempenho sofrem algumas alterações orgânicas. Nesse contexto, as formas de organização do trabalho que não levam em conta a variabilidade do indivíduo, podem aumentar o risco de repercussões prejudiciais à saúde do mesmo ${ }^{(34)}$.

Estudo sobre enfermeiras de um hospital público em Salvador - BA revelou, baseado em autoavaliação dos participantes que, comparando-se as enfermeiras do turno noturno com o diurno, as primeiras apresentaram taxas mais elevadas de prevalência de sintomas e problemas de saúde. Os sintomas associados ao trabalho noturno foram: alteração da qualidade de sono, distúrbios digestivos e fadiga ${ }^{(35)}$.

Dentre os trabalhadores com DM, é importante ressaltar que, aproximadamente, metade deles realizava plantões noturnos. A redução da quantidade de profissionais, a continuidade dos procedimentos terapêuticos aos clientes, as intercorrências, o sono e a fadiga inerentes a esse turno de trabalho constituem um contexto que pode interferir na prevenção e controle da doença ${ }^{(35)}$.

\section{CONCLUSÃO}

A prevalência de DM autorreferida em trabalhadores de enfermagem do Hospital São Paulo encontrada no presente estudo foi de 3\%, sendo menor aos valores referidos entre a população da cidade de São Paulo. Entre os fatores relacionados, como sendo de risco para desenvolver a doença, encontraram-se idade, sexo, sobrepeso e história familiar. Dentre estes, o excesso de peso é o único fator modificável, portanto, deve-se considerar a importância de uma alimentação correta, assim como a prática de atividade física por esses profissionais. Estes fatores implicam em aumento da qualidade de vida e, consequentemente, maior produtividade e qualidade do trabalho realizado, o que de certo modo refletiria proporcionalmente na melhora do cuidado prestado aos clientes atendidos no dia a dia por esses profissionais.

$\mathrm{O}$ fato da maioria dos funcionários com DM tipo 2 não seguirem a dieta adequada deve ser considerado. É relevante investigar os motivos que desencadeiam tal 
atitude, para que então possam ser discutidas medidas que favoreçam o estado nutricional dos funcionários.

A maioria dos profissionais com DM trabalhava em plantões noturnos. Este dado chama a atenção pelo fato de ser o plantão mais extenso e, portanto, mais cansativo. Além disso, o metabolismo de quem não dorme à noite, é alterado, o que poderia ser mais um fator prejudicial para

\section{REFERÊNCIAS}

1. Duncan BB, Schmidt MI, Polanczyk CA, Homrich CS, Rosa RS, Achutti AC. Fatores de risco para doenças não transmissíveis em áreas metropolitanas na região sul do Brasil: prevalência e simultaneidade. Rev Saúde Pública $=\mathrm{J}$ Public Health. 1993;27(1):43-8.

2. Mariath AB, Grillo LP, Silva RO, Schmitz P, Campos IC, Medina JRP, Kruger RM. Obesidade e fatores de risco para o desenvolvimento de doenças crônicas não transmissíveis entre usuários de unidade de alimentação e nutrição. Cad Saúde Pública = Rep Public Health. 2007;23(4):897-905.

3. Lyra R, Oliveira M, Lins D, Cavalcanti N. Prevenção do diabetes mellitus tipo 2. Arq Bras Endocrinol Metab. 2006;50(2):239-49.

4. Alberti KG, Zimmet P, Shaw J. International Diabetes Federation: a consensus on Type 2 diabetes prevention. Diabet Med. 2007;24(5):451-63.

5. Tuomilehto J, Lindström J, Eriksson JG, Valle TT, Hämäläinen H, Ilanne-Parikka P, Keinänen-Kiukaanniemi S, Laakso M, Louheranta A, Rastas M, Salminen V, Uusitupa M; Finnish Diabetes Prevention Study Group. Prevention of type 2 diabetes mellitus by changes in lifestyle among subjects with impaired glucose tolerance. N Engl J Med. 2001;344(18):1343-50. Comment in: N Engl J Med. 2001;345(9):696; author reply 696-7. BMJ. 2001;323(7319):997. ACP J Club. 2001;135(3):101. N Engl J Med. 2001;344(18):1390-2.

6. Brasil. Ministério da Saúde. Secretaria de Políticas de Saúde. Departamento de Ações Programáticas Estratégicas. Plano de reorganização da atenção à hipertensão arterial e ao Diabetes Mellitus: manual de hipertensão arterial e Diabetes Mellitus. Brasília: Ministério da Saúde; 2001.

7. Brasil, Ministério da Saúde; Organização Pan-Americana da Saúde. Avaliação do Plano de Reorganização da Atenção à Hipertensão Arterial e ao Diabetes Mellitus no Brasil. Brasilia: Ministério da Saúde; 2004.

8. Watson J. Nursing: human science and human care. A theory of nursing. NLN Publ. 1988;(15-2236):1-104.

9. Vieira ABD, Alves ED, Kamada I. Cuidando do cuidador: percepções e concepções de auxiliares de enfermagem acerca do cuidado de si. Texto \& Contexto Enferm. 2007;16(1):1525.

10. Silva DMPP, Marziale MHP. Absenteísmo de trabalhadores de enfermagem em um hospital universitário. Rev Latinoam Enferm. 2000;8(5):44-51.

11. Murofuse NT, Abranches SS, Napoleão AA. Reflexões sobre estresse e Burnout e a relação com a enfermagem. Rev Latinoam Enferm. 2005;13(2):255-61.

12. Farias SNP, Mauro MYC, Zeitoune RCG. Questões legais sobre a saúde do trabalhador de enfermagem. Rev Enferm UERJ. 2000;8(1):28-32.

13. World Health Organization. Obesity: preventing and managing the global epidemic; report of a WHO consultation on obesity. Geneva: World Health Organization; 1998.

14. Goldenberg P, Schenkman S, Franco LJ. Prevalência de os portadores de DM do que para indivíduos saudáveis.

\section{AGRADECIMENTO}

Agradecemos aos funcionários que colaboraram participando deste estudo, assim como o apoio financeiro fornecido pelo PIBIC/CNPq.

Diabetes mellitus: diferenças de gênero e igualdade entre os sexos. Rev Bras Epidemiol. 2003;6(1):18-28.

15. King H, Rewers M. Global estimates of diabetes mellitus and impaired glucose tolerance in adults. WHO Ad Hoc Diabetes Reporting Group. Diabetes Care. 1993;16(1):15777. Comment in: Diabetes Care. 1993;16(11):1530.

16. Goldenberg P, Franco LJ, Pagliaro H, Silva RS, Santos CA. Diabetes mellitus auto-referido no município de São Paulo: prevalência e desigualdade. Cad Saúde Pública $=$ Rep Public Health. 1996;12(1):37-45.

17. Brasil. Ministério da Saúde. Departamento de Atenção Básica. Área Técnica de Diabetes e Hipertensão Arterial. Hipertensão arterial sistêmica (HAS) e Diabetes mellitus (DM): protocolo. Brasília: Ministério da Saúde; 2001.96 p.

18. American Diabetes Association. Standards of Medical Care in Diabetes. American Diabetes Association: Clinical Practice Recommendations 2004: Position Statement. Diabetes Care. 2004;27(Suppl 1):S15-35.

19. Torquato MTCG, Montenegro RM, Viana RAHG, et al. Estudo de prevalência do diabetes melito e intolerância à glicose na população urbana de 30 a 69, no município de Ribeirão Preto. Arq Bras Endocrinol Metab. 1999; 43(Supl 1):S190.

20. Gomes MB, Giannella Neto D, Mendonça E, Tambascia MA, Fonseca RM, Réa RR, et al. Prevalência de sobrepeso e obesidade em pacientes com diabetes mellitus do tipo 2 no Brasil: estudo multicêntrico nacional. Arq Bras Endocrinol Metab. 2006;50(1):136-44.

21. Mackay MF, Haffner SM, Wagenknecht LE, D'Agostino RB Jr, Hanley AJ. Prediction of type 2 diabetes using alternate anthropometric measures in a multi-ethnic cohort: the insulin resistance atherosclerosis study. Diabetes Care. 2009;32(5):956-8.

22. Shimokata H, Muller DC, Andres R. Studies in the distribution of body fat. III. Effects of cigarette smoking. JAMA.1989;261(8):1169-73. Comment in: JAMA. 1989;262(9):1185-6.

23. Frati AC, Iniestra F, Ariza CR. Acute effect of cigarette smoking on glucose tolerance and other cardiovascular risk factors. Diabetes Care. 1996;19(2):112-8.

24. Janzon L, Berntorp K, Hanson M, Lindell SE, Trell E. Glucose tolerance and smoking: a population study of oral and intravenous glucose tolerance tests in middle-aged men. Diabetologia. 1983;25(2):86-8.

25. Uchimoto S, Tsumura K, Hayashi T, Suematsu C, Endo G, Fujii S, Okada K. Impact of cigarette smoking on the incidence of Type 2 diabetes mellitus in middle-aged Japanese men: the Osaka Health Survey. Diabet Med. 1999;16(11):951-5.

26. Sladek R, Rocheleau G, Rung J, Dina C, Shen L, Serre D, et al. A genome-wide association study identifies novel risk loci for type 2 diabetes. Nature. 2007;445(7130):881-5.

27. Hariri S, Yoon PW, Qureshi N, Valdez R, Scheuner MT, Khoury MJ. Family history of type 2 diabetes: a populationbased screening tool for prevention? Genet Med. 
2006;8(2):102-8.

28. Teixeira RC, Mantovani MF. Enfermeiros com doença crônica: as relações com o adoecimento, a prevenção e o processo de trabalho. Rev Esc Enferm USP. 2009;43(2):415-21.

29. Gross LJ, Silveiro SP, Camargo JL, Reichelt AJ, Azevedo MJ. Diabetes melito: diagnóstico, classificação e avaliação do controle glicêmico. Arq Bras Endocrinol Metab. 2002;46(1):16-26.

30. Santos MA, Zanetti ML. Dificuldades dos pacientes diabéticos para o controle da doença: sentimentos e comportamentos. Rev Latinoam Enferm. 2007;15(6):110512.

31. Gamba MA. A importância da assistência de enfermagem na prevenção, controle e avaliação à pacientes portadores de diabetes com neuropatia e vasculopatia. Acta Paul Enferm. 1991;4(2/4):7-19.
32. Zanetti ML, Mendes IAC. Tendência do locus de controle de pessoas diabéticas. Rev Esc Enferm USP. 1993;27(2):24662.

33. Zanetti ML, Otero LM, Peres DS, Santos MA, Guimarães FPM, Freitas MCF. Progress of the patients with diabetes mellitus who were managed with the staged diabetes management framework. Acta Paul Enferm. 2007;20(3):33844.

34. Lisboa MTL, Oliveira MM, Reis LD. O trabalho noturno e a prática de enfermagem: uma percepção dos estudantes de enfermagem. Esc Anna Nery Rev Enferm. 2006;10(3):3938.

35. Fischer MF, Teixeira LR, Borges FNS, Gonçalves MBL, Ferreira RM. Percepção de sono: duração, qualidade e alerta em profissionais da área de enfermagem. Cad Saúde Pública $=$ Rep Public Health. 2002;18(5):1261-9. 